\title{
Changes in Corneal Morphology with Age in Asian Population: A Multicenter Study of 30,618 Cases
}

\author{
Ruijue Ma $\cdot$ Yuanyuan Liu $\cdot$ Lin Zhang $\cdot$ Jiaonan Ma \\ Tong Cui · Yulin Lei · Jie Hou · Zhengwei Shen · Xianglong Yi • \\ Gang Liang • Yan Wang (D)
}

Received: July 5, 2021 / Accepted: September 14, 2021 / Published online: October 10, 2021

(C) The Author(s) 2021

\begin{abstract}
Introduction: To evaluate normal reference ranges for corneal morphological parameters and investigate age-related changes in these parameters in Asian subjects with healthy eyes in order to provide reference data for preoperative evaluation of corneal refractive surgery and the early differential diagnosis of subclinical and asymptomatic keratoconus.

Methods: This cross-sectional, multicenter, observational study was conducted in five pro-
\end{abstract}

Ruijue Ma and Yuanyuan Liu contributed equally to the work presented and should therefore be regarded as equivalent authors.

R. Ma $\cdot$ L. Zhang $\cdot$ J. Ma $\cdot$ Y. Wang $(\bowtie)$

Tianjin Eye Institute, Tianjin Key Lab of

Ophthalmology and Visual Science, Tianjin Eye

Hospital, Nankai University Affiliated Eye Hospital,

No 4. Gansu Road, He-ping District, Tianjin 300020,

China

e-mail: wangyan7143@vip.sina.com

Y. Liu

Department of Health Statistics, College of Public

Health, Tianjin Medical University, Tianjin, China

T. Cui

Department of Ophthalmology, Shanxi Eye

Hospital, Shanxi, China vinces of China, from January 2014 through October 2019. It is a retrospective analysis. Examiner-blinded clinical measurements were performed after stratification of the subjects into the following age groups: $<18,18-30$, $31-40,41-50$. We evaluated 30,618 healthy eyes of Chinese subjects who exhibited a normal corneal morphology, had no history of eye surgery or trauma, stopped wearing soft contact lenses for at least 2 weeks (rigid contact lenses for at least 4 weeks), and underwent topographic studies for both eyes on the same day.

Results: While the anterior and posterior corneal curvatures (K1 and K2) increased with age, corneal astigmatism of the anterior and posterior surfaces $(\Delta \mathrm{K})$ and central, minimum, and

\section{Y. Lei $\cdot$ J. Hou}

Department of Ophthalmology, Jinan Mingshui Eye Hospital, Shandong, China

\section{Z. Shen}

Ophthalmic Department, Wuhan General Hospital of Guangzhou Military Command of PLA, Hubei, China

\section{Yi}

Department of Ophthalmology, The First Affiliated Hospital of Xinjiang Medical University, Xinjiang, China

G. Liang

Department of Ophthalmology, Red Cross Hospital, Yunnan, China 
overall corneal thicknesses decreased with age. Age-related decrease of the overall corneal thickness was more obvious toward the periphery. The anterior and posterior corneal surface heights exhibited a decrease and an increase, respectively. Both index of height asymmetry (IHA) and index of vertical asymmetry (IVA) exhibited an increase with age.

Conclusions: The cornea exhibits overall thinning with age and gradually changes from a flat ellipse to an elongated ellipse in Asian individuals with healthy eyes. However, the anterior and posterior surfaces become smoother with age. Owing to the very large number of cases, these small differences are statistically significant. The results obtained are consistent with the hypothesis that a normal cornea seems to withstand quite well the effect of IOP, external pressures, and the natural cross-linking.

Keywords: Corneal curvatures; Corneal morphology; Corneal thickness; Keratoconus; Multicenter study

\section{Key Summary Points}

Why carry out this study?

Accurate assessment of the range of normal corneal morphological parameters is crucial for the identification of many preclinical corneal diseases.

With the rapid development of technology for corneal refractive surgery, the effective prevention of postoperative corneal dilation and increased safety has become an important part of preoperative screening for individuals scheduled.

A defined normal reference range for an evaluation index is an important prerequisite for the correct assessment of examination findings. The normal values of corneal morphology vary among different populations, so we designed this multicenter study for Asians.

\section{What was learned from the study?}

While the anterior and posterior corneal curvatures (K1 and K2) increased with age, corneal astigmatism of the anterior and posterior surfaces $(\Delta \mathrm{K})$ and central, minimum, and overall corneal thicknesses decreased with age. Age-related decrease of the overall corneal thickness was more obvious toward the periphery. The anterior and posterior corneal surface heights exhibited a decrease and an increase, respectively. Both index of height asymmetry (IHA) and index of vertical asymmetry (IVA) exhibited an increase with age.

The cornea exhibits overall thinning with age and gradually changes from a flat ellipse to an elongated ellipse in Asian individuals with healthy eyes. However, the anterior and posterior surfaces become smoother with age. Therefore, with the increase in age, normal corneal morphology shows a gradual pseudoprotuberance state.

\section{INTRODUCTION}

Accurate assessment of the range of normal corneal morphological parameters is crucial for the identification of many preclinical corneal diseases, among which keratoconus is closely related to corneal morphological changes. Keratoconus is a chronic progressive disease without inflammation, characterized by progressive thinning and bulging of the normally round cornea into a cone-like shape. It can cause irregular myopic astigmatism and reduce the corrected visual acuity by varying degrees. Keratoconus mostly occurs in adolescents and gradually progresses until the age of 20 or 30 years. A minority of patients are diagnosed after the age of 40 years, following which the condition slowly progresses [1-5]. Keratoconus 
is generally bilateral, although it may occur successively in each eye [5-7]. The disease has a certain familial hereditary tendency, although there is no obvious sex-related difference [8]. All ethnic groups can exhibit this condition, with the incidence ranging from $0.05 \%$ to $0.23 \%$ [9-11].

Clinical keratoconus can be easily diagnosed on the basis of obvious corneal morphological changes such as Vogt striae, Fleischer rings, corneal protuberance, and even corneal scarring $[11,12]$. Moreover, experts have agreed that abnormal posterior ectasia, abnormal distribution of the corneal thickness, and clinical noninflammatory corneal thinning are mandatory findings for a diagnosis of keratoconus [12]. However, it is very difficult to diagnose subclinical and early asymptomatic keratoconus [13]. In recent years, with the rapid development of technology for corneal refractive surgery, there is a rapid increase in the number of individuals receiving this surgery. Therefore, timely detection of subclinical and early asymptomatic keratoconus for the effective prevention of postoperative corneal ectasia and increased safety $[14,15]$ has become an important part of preoperative screening for individuals scheduled for such surgeries.

Accurate detection of minute changes in the corneal morphology, such as the corneal curvature, corneal thickness, and anterior and posterior corneal surface heights, among others, is necessary for differentiating subclinical and early asymptomatic keratoconus from normal age-related changes. It is well known that a defined normal reference range for an evaluation index is an important prerequisite for the correct assessment of examination findings. Therefore, we designed the present large-scale, multicenter study involving Asian subjects with healthy eyes, with the aim of evaluating normal reference ranges for corneal morphological parameters and investigating age-related changes in order to provide reference data that can aid in the differentiation of normal age-related changes from the changes seen in subclinical and early asymptomatic keratoconus.

\section{METHODS}

\section{Study Subjects}

This cross-sectional study was conducted in five medical centers in different regions of China: Tianjin Eye Hospital, Jinan Mingshui Eye Hospital in Shandong province, Wuhan General Hospital of Guangzhou Military Command of PLA in Hubei province, The First Affiliated Hospital of Xinjiang Medical University in Xinjiang province, and Red Cross Hospital in Yunnan province. This is a retrospective analysis. The study protocol adhered to the tenets of the Declaration of Helsinki. Ethical approval was not required because this retrospective study did not deal with human tissue samples, and there were no concerns about privacy.

We collected anterior segment examination data from January 2014 through October 2019. For an initial set of more than 48,056 samples, we performed screening according to the following inclusion criteria: patients aged $10-50$ years, which is the range for the occurrence of keratoconus [1-3]; those who underwent corneal topography studies for both eyes on the same day; those who exhibited a normal corneal morphology without any corneal macula; those without a history of eye surgery or trauma; and those who had stopped wearing soft contact lenses for at least 2 weeks or rigid contact lenses for at least 4 weeks. Eyes with keratoconus, suspected keratoconus, or subclinical keratoconus diagnosed according to a global consensus [12] and the ABCD Grading System for keratoconus [16] were excluded. The enrolled patients were divided into the following four groups according to their age in years: $<18,18-30,31-40$, and 41-50. All measured parameters were compared among these age groups.

\section{Clinical Examinations}

We evaluated the anterior and posterior corneal surface curvatures K1and $\mathrm{K} 2$; astigmatism of the anterior and posterior corneal surfaces $(\Delta \mathrm{K}$, defined as the absolute difference between readings in the $\mathrm{K} 1$ and $\mathrm{K} 2$ meridians [17]); 
central, minimum, and overall corneal thicknesses; anterior and posterior corneal surface heights at the thinnest points; and corneal morphological parameters closely associated with keratoconus, namely the index of height asymmetry (IHA) and index of vertical asymmetry (IVA). IHA represents the degree of symmetry in the horizontal vertex height data relative to the reflection axis, while IVA represents the degree of symmetry in the corneal radius at the horizontal apex relative to the reflection axis. IHA and IVA values that are higher than normal suggest an increased risk of keratoconus.

We used an examiner-blinded method for all measurements, which were performed using Pentacam (three-dimensional anterior segment analysis and diagnostic system; OCULUS, Germany). The five research centers used the same device (Pentacam HR) for measurements and data collection. All equipment are calibrated by engineers of the same company every 3 months. The calibration tool is a standard simulated eye with fixed thickness, curvature, and grayscale parameters. Using the optical principle for evaluation of the simulated eye, the device records standard parameters for the simulated eye into its digital signal processor in order to ensure appropriate calibration and instrument precision.

We performed measurements under natural pupil conditions in a darkroom. After adjusting the mandible in the mandibular pad, we asked the subjects to keep their eyes open and watch the circles in the blue-ribbon indicator after blinking twice. The examiner (a member of the research team) used the joystick to aim and focus with the screen cue. Detection bias was avoided by selection of the automatic measuring system release mode; automatic measurement began when the instrument detected the pupil center, edge, and clear corneal apex. According to quality specification requirements, the instrument only displays the test results marked as "OK" to the examiner.

\section{Statistical Analysis}

All data were analyzed using SAS 9.4 (SAS Institute Inc., Cary, NC, USA). Quantitative data are described as means \pm standard deviations if data meet normal distribution, otherwise medians (M) (Q1-Q3) were used. For comparisons between two groups, the independent samples $t$ test and the Wilcoxon rank sum test were applied for normally and non-normally distributed data, respectively. The Kruskal-Wallis test was used for comparisons among three or more groups for non-normally distributed data. Spearman's rank correlation analysis was used to test for trends for nonnormally distributed data. $P$ values of less than 0.05 were considered statistically significant.

\section{RESULTS}

In total, 30,618 healthy eyes of patients aged $10-50$ years [mean age, 25.00 (20.00, 31.00) years] and residing in Tianjin, Shandong, Hubei, Xinjiang, or Yunnan were included (Fig. 1).

\section{Corneal Curvature}

The anterior and posterior corneal curvatures (K1 and K2) exhibited significant differences among the age groups $(P<0.0001)$ and increased with age (anterior: $r_{\mathrm{s}, \mathrm{K} 1}=0.16124$, $r_{\mathrm{s}, \mathrm{K} 2}=0.12646, \quad P<0.0001 ;$ posterior: $r_{\mathrm{s}, \mathrm{K} 1}=$ $-0.10820, \quad r_{\mathrm{s}, \mathrm{K} 2}=-0.03978, \quad P<0.0001$; Table 1 and Fig. 2). Astigmatism of the anterior and posterior surfaces also showed significant differences among the age groups $(P<0.0001)$, although it tended to decrease with age $\left(r_{\mathrm{s}, \text { anterior }}=-0.06914, \quad r_{\mathrm{s}, \text { posterior }}=-0.10331\right.$, $P<0.0001$; Table 1 and Fig. 2).

\section{Corneal Thickness}

Both the central and thinnest corneal thicknesses showed significant differences among the age groups $(P<0.0001)$, with both exhibiting a tendency to decrease with age $\left(r_{\mathrm{s}, \mathrm{CCT}}=\right.$ $\left.-0.03571, \quad r_{\mathrm{s}, \mathrm{TCT}}=-0.03580, \quad P<0.0001\right)$. There was no correlation between the difference in the two parameters and age $\left(r_{\mathrm{s}}=-0.00550\right.$, $P=0.3362$; Table 2 and Fig. 3 ). 


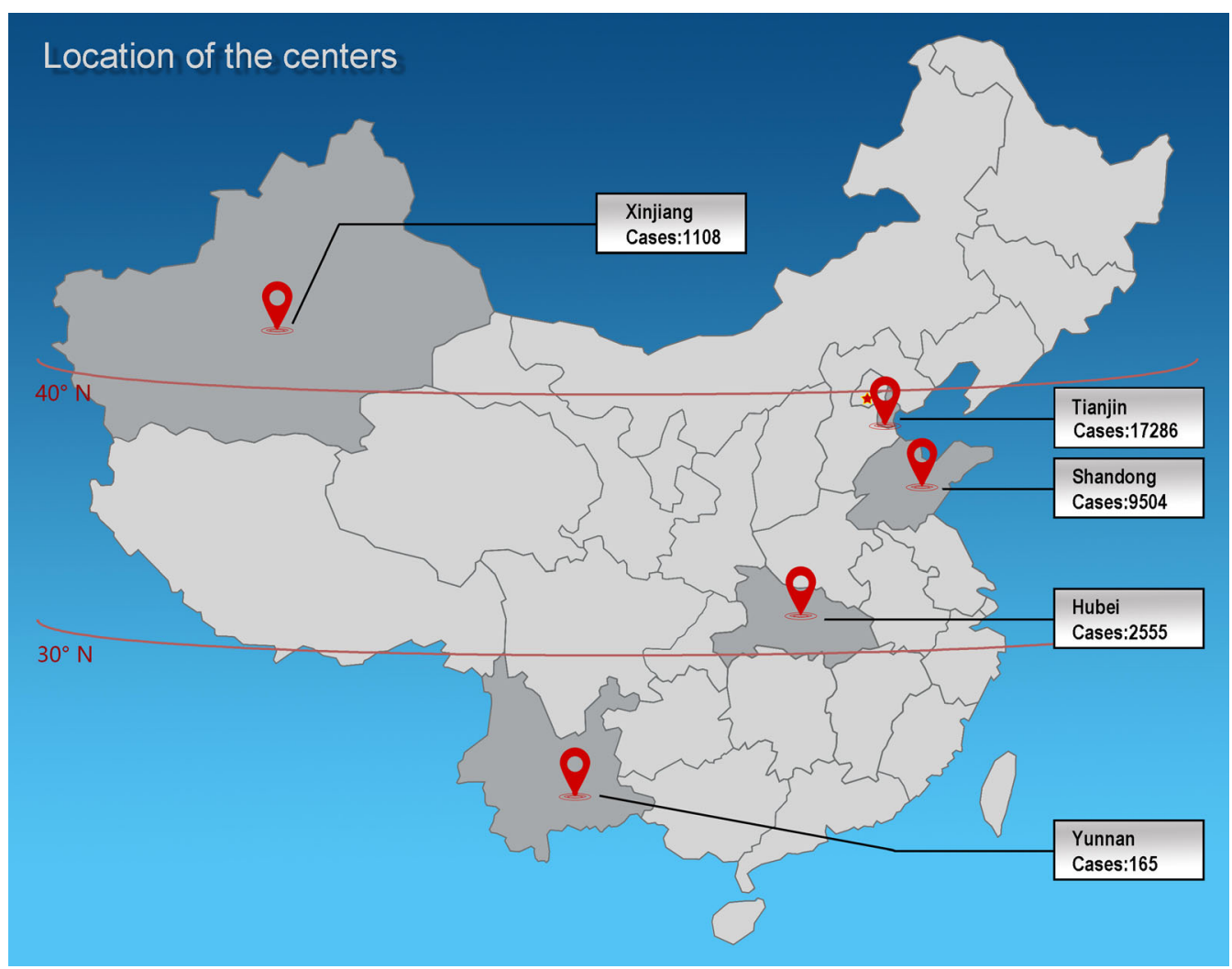

Fig. 1 Locations of provinces and research centers included in the present study, with the number of subjects enrolled in each center. We investigated age-related

We also analyzed the corneal thickness spatial profile (CTSP) for diameters from 0 to $10 \mathrm{~mm}$. For this measurement, concentric circles are made with the thinnest point of the cornea as the center, and the circumference average thickness is calculated for the different diameters. We found that the cornea exhibited overall thinning with age, with the decrease becoming more obvious toward the periphery (Table 3 and Fig. 3).

\section{Corneal Surface Height}

There were significant differences in the anterior and posterior corneal surface heights among the different age groups $(P<0.0001)$. While the anterior corneal surface height was negatively correlated with age, thus tending to decrease with age $\left(r_{\mathrm{s}}=-0.02522, P<0.0001\right)$, the posterior corneal surface height was positively correlated with age, thus tending to changes in corneal morphological parameters associated with keratoconus in Chinese subjects with healthy eyes

increase with age $\left(r_{\mathrm{s}}=0.05597, P<0.0001\right.$; Table 4 and Fig. 4).

\section{Corneal Morphological Parameters}

Both IHA and IVA were significantly different among the age groups $\left(P_{\mathrm{IHA}}<0.0001, P_{\mathrm{IVA}}=\right.$ 0.0166), with both parameters exhibiting an increase with age $\left(r_{\mathrm{s}, \mathrm{IHA}}=0.03418, P<0.0001\right.$; $r_{\mathrm{s}, \mathrm{IVA}}=0.01599, P=0.0051$; Table 5 and Fig. 4 ).

\section{DISCUSSION}

In the present study, we determined age-related changes in corneal morphological parameters in a sample of Asian subjects with healthy eyes in the age range of $10-50$ years, which is the predominant age range for the occurrence of keratoconus. 
Table 1 Anterior and posterior corneal curvatures and astigmatism values for Chinese subjects with healthy eyes stratified into different age groups

\begin{tabular}{|c|c|c|c|c|c|}
\hline Age groups & $N$ (missing) & Mean \pm SD & $M(Q 1-Q 3)$ & $\chi^{2}$ & $P$ \\
\hline \multicolumn{6}{|c|}{ Anterior corneal curvature } \\
\hline \multicolumn{6}{|l|}{ K1 } \\
\hline$<18$ years $*$ & $780(0)$ & $42.29 \pm 1.29$ & $42.25(41.50-43.10)$ & \multirow[t]{4}{*}{799.0081} & \multirow[t]{4}{*}{$<0.0001$} \\
\hline $18-30$ years ${ }^{\triangle}$ & $21,495(0)$ & $42.57 \pm 1.30$ & $42.60(41.70-43.40)$ & & \\
\hline $31-40$ years $^{\#}$ & $6945(0)$ & $43.02 \pm 1.31$ & $43.00(42.20-43.90)$ & & \\
\hline $41-50$ years $^{\#}$ & $1398(0)$ & $43.11 \pm 1.33$ & $43.20(42.20-44.00)$ & & \\
\hline \multicolumn{6}{|l|}{ K2 } \\
\hline$<18$ years $^{*}$ & $780(0)$ & $43.41 \pm 1.41$ & $43.40(42.50-44.40)$ & \multirow[t]{4}{*}{489.8542} & \multirow[t]{4}{*}{$<0.0001$} \\
\hline $18-30$ years $^{\triangle}$ & $21,495(0)$ & $43.71 \pm 1.43$ & $43.70(42.70-44.70)$ & & \\
\hline $31-40$ years $^{\#}$ & $6945(0)$ & $44.08 \pm 1.43$ & $44.10(43.10-45.00)$ & & \\
\hline $41-50$ years $^{\#}$ & $1398(0)$ & $44.16 \pm 1.42$ & $44.20(43.10-45.10)$ & & \\
\hline \multicolumn{6}{|c|}{ Astigmatism $(\Delta \mathrm{K})$} \\
\hline$<18$ years $^{*}$ & $780(0)$ & $1.11 \pm 0.65$ & $1.00(0.70-1.40)$ & \multirow[t]{4}{*}{159.6708} & \multirow[t]{4}{*}{$<0.0001$} \\
\hline $18-30$ years $^{*}$ & $21,495(0)$ & $1.13 \pm 0.67$ & $1.00(0.70-1.50)$ & & \\
\hline $31-40$ years ${ }^{\triangle}$ & $6945(0)$ & $1.06 \pm 0.73$ & $0.90(0.60-1.40)$ & & \\
\hline $41-50$ years ${ }^{\triangle}$ & $1398(0)$ & $1.05 \pm 0.74$ & $0.90(0.50-1.40)$ & & \\
\hline \multicolumn{6}{|c|}{ Posterior corneal curvature } \\
\hline \multicolumn{6}{|l|}{ K1 } \\
\hline$<18$ years ${ }^{*}$ & $780(0)$ & $-6.01 \pm 0.21$ & $-6.00(-6.20$ to -5.90$)$ & \multirow[t]{4}{*}{368.1386} & \multirow[t]{4}{*}{$<0.0001$} \\
\hline $18-30$ years $^{\triangleleft}$ & $21,495(0)$ & $-6.07 \pm 0.21$ & $-6.10(-6.20$ to -5.90$)$ & & \\
\hline $31-40$ years $^{\#}$ & $6945(0)$ & $-6.12 \pm 0.22$ & $-6.10(-6.30$ to -6.00$)$ & & \\
\hline $41-50$ years $^{\S}$ & $1398(0)$ & $-6.13 \pm 0.23$ & $-6.10(-6.30$ to -6.00$)$ & & \\
\hline \multicolumn{6}{|c|}{ 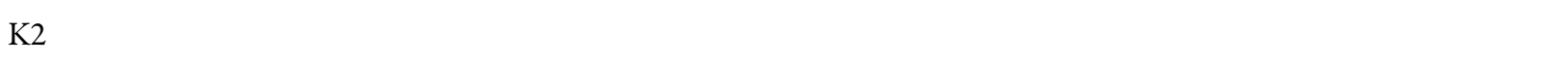 } \\
\hline$<18$ years $^{*}$ & $780(0)$ & $-6.38 \pm 0.25$ & $-6.40(-6.50$ to -6.20$)$ & \multirow[t]{4}{*}{57.8636} & \multirow[t]{4}{*}{$<0.0001$} \\
\hline $18-30$ years $^{\triangle}$ & $21,495(0)$ & $-6.42 \pm 0.25$ & $-6.40(-6.60$ to -6.30$)$ & & \\
\hline $31-40$ years ${ }^{\triangle}$ & $6945(0)$ & $-6.44 \pm 0.25$ & $-6.40(-6.60$ to -6.30$)$ & & \\
\hline $41-50$ years ${ }^{\triangle}$ & $1398(0)$ & $-6.44 \pm 0.26$ & $-6.40(-6.60$ to -6.30$)$ & & \\
\hline \multicolumn{6}{|c|}{ Astigmatism $(\Delta \mathrm{K})$} \\
\hline$<18$ years $^{*}$ & $780(0)$ & $0.37 \pm 0.14$ & $0.40(0.30-0.50)$ & \multirow[t]{3}{*}{339.6934} & \multirow[t]{3}{*}{$<0.0001$} \\
\hline $18-30$ years $^{\triangle}$ & $21,495(0)$ & $0.35 \pm 0.14$ & $0.30(0.30-0.40)$ & & \\
\hline $31-40$ years ${ }^{\#}$ & $6945(0)$ & $0.33 \pm 0.15$ & $0.30(0.20-0.40)$ & & \\
\hline
\end{tabular}


Table 1 continued

\begin{tabular}{llclll}
\hline Age groups & $\boldsymbol{N}$ (missing) & Mean \pm SD & M (Q1-Q3) & $\chi^{2}$ & $P$ \\
\hline $41-50$ years $^{\S}$ & $1398(0)$ & $0.30 \pm 0.16$ & $0.30(0.20-0.40)$ & & \\
\hline
\end{tabular}

Wilcoxon rank sum test

Different symbols $\left(\triangle,{ }^{*}, \#, \S\right)$ indicate significant differences between groups

$S D$ standard deviation, $M$ median

(A)Anterior Surface Curvature

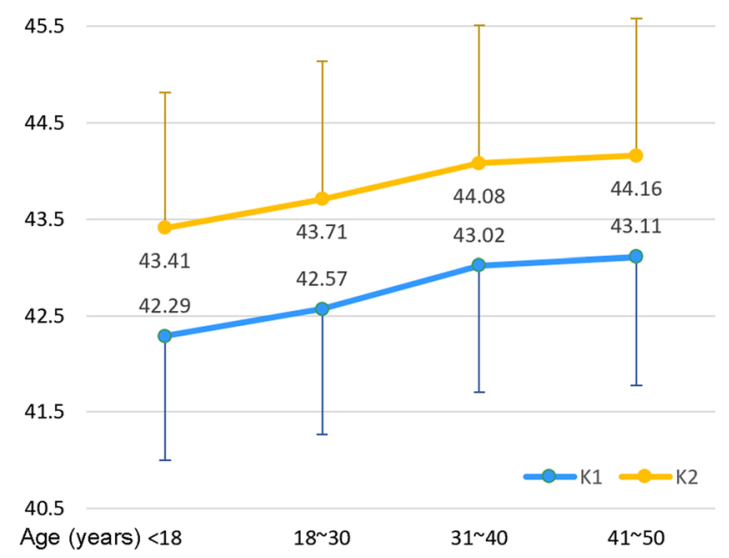

(C) Anterior corneal astigmatism $(\triangle K)$

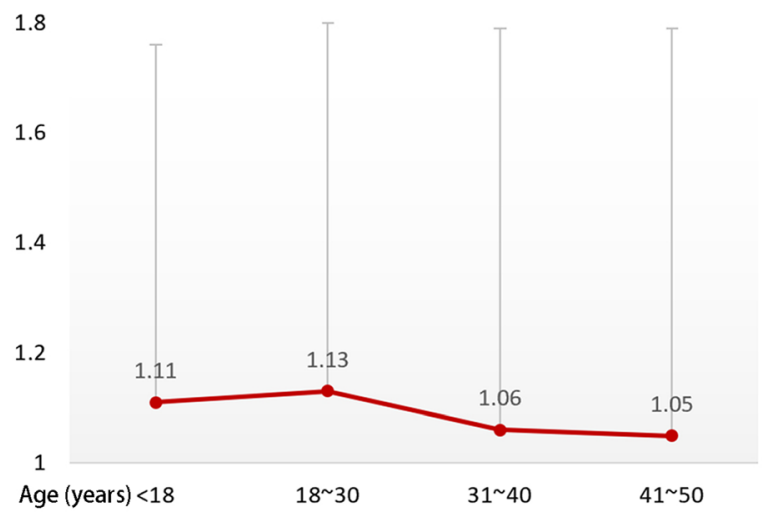

Fig. 2 Age-related changes in the corneal curvature and astigmatism $(\Delta \mathrm{K})$ in Chinese subjects with healthy eyes. a The anterior corneal curvature (K1 and K2) exhibits an increase with age. $\mathbf{b}$ The posterior corneal curvature (K1

With the rapid increase in the number of refractive surgeries performed worldwide, corneal ectasia after refractive surgery has become a common concern for refractive surgery and corneal experts. Subclinical and early
(B) Posterior Surface Curvature

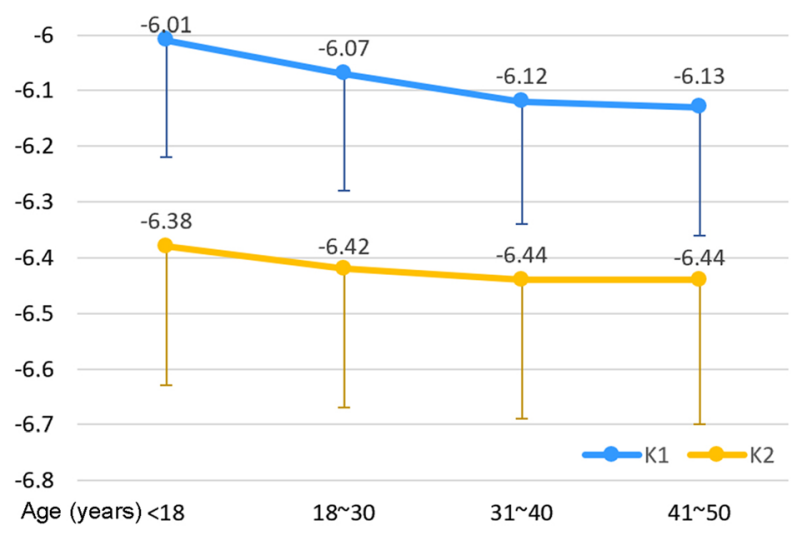

(D) Posterior corneal astigmatism $(\triangle \mathrm{K})$

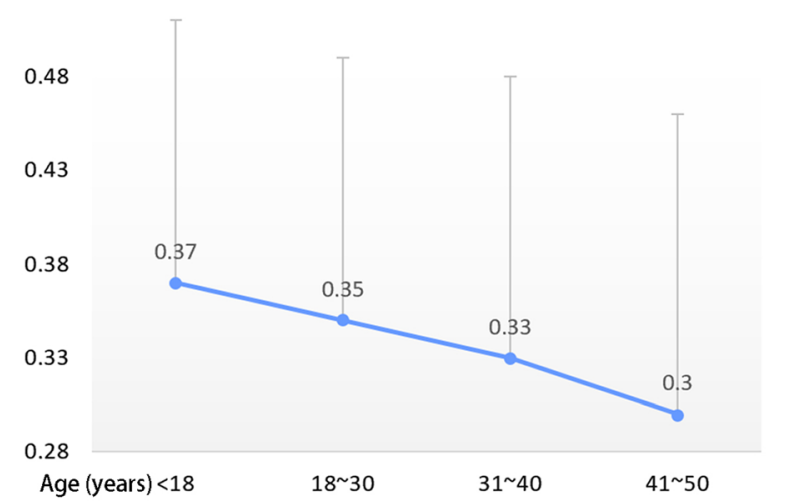

and K2) also exhibits an increase in magnitude with age. c Anterior corneal surface astigmatism exhibits a decrease with age. d Posterior corneal surface astigmatism also exhibits a decrease with age

asymptomatic keratoconus that remains undiagnosed before surgery is the main cause of this postoperative corneal ectasia, which leads to an irreversible decrease in the corrected visual acuity after surgery. Therefore, even though the 
Table 2 Corneal thickness values for Chinese subjects with healthy eyes stratified into different age groups

\begin{tabular}{|c|c|c|c|c|c|}
\hline Age groups & $N$ (missing) & Mean \pm SD & $M(Q 1-Q 3)$ & $\chi^{2}$ & $P$ \\
\hline \multicolumn{6}{|c|}{ Central corneal thickness } \\
\hline$<18$ years $^{*}$ & $780(1)$ & $544.73 \pm 30.33$ & $544.00(522.00-563.00)$ & 40.5549 & $<0.0001$ \\
\hline $18-30$ years* $^{*}$ & $21,495(9)$ & $543.78 \pm 29.40$ & $543.00(524.00-563.00)$ & & \\
\hline $31-40$ years $^{\triangle}$ & $6945(0)$ & $541.54 \pm 28.63$ & $541.00(522.00-560.00)$ & & \\
\hline $41-50$ years $^{\triangle}$ & $1398(0)$ & $540.54 \pm 29.07$ & $540.00(520.00-561.00)$ & & \\
\hline \multicolumn{6}{|c|}{ Minimum corneal thickness } \\
\hline$<18$ years $^{*}$ & $780(1)$ & $541.53 \pm 30.18$ & $540.00(519.00-560.00)$ & 41.7169 & $<0.0001$ \\
\hline $18-30$ years* $^{*}$ & $21,495(9)$ & $540.76 \pm 29.24$ & $540.00(521.00-560.00)$ & & \\
\hline $31-40$ years $^{\triangle}$ & $6945(0)$ & $538.58 \pm 28.52$ & $538.00(519.00-557.00)$ & & \\
\hline $41-50$ years ${ }^{\triangle}$ & $1398(0)$ & $537.24 \pm 28.74$ & $536.50(516.00-557.00)$ & & \\
\hline
\end{tabular}

Wilcoxon rank sum test

Different symbols $\left(\triangle,{ }^{*}\right)$ indicate significant differences between groups

$S D$ standard deviation, $M$ median

(A) Corneal Thickness in Different Ages

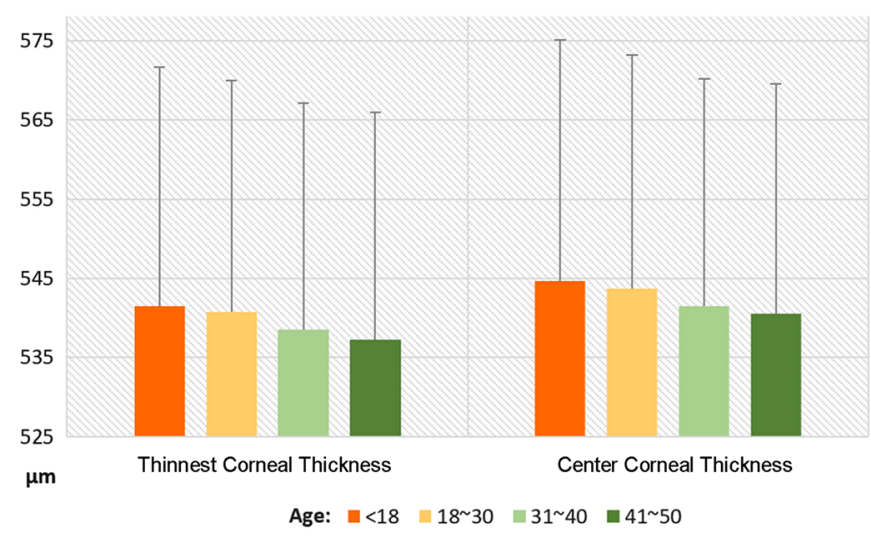

Fig. 3 Age-related trends in the corneal thickness in Chinese subjects with healthy eyes. a Both the central corneal thickness and the minimum corneal thickness exhibit a decrease with age. $\mathbf{b}$ There is overall corneal

incidence of this complication is low, it has detrimental consequences and should be prevented.

The differential diagnosis of subclinical and early asymptomatic keratoconus remains a worldwide problem. Corneal topography is considered the gold standard for the diagnosis
(B) CTSP in Different Ages

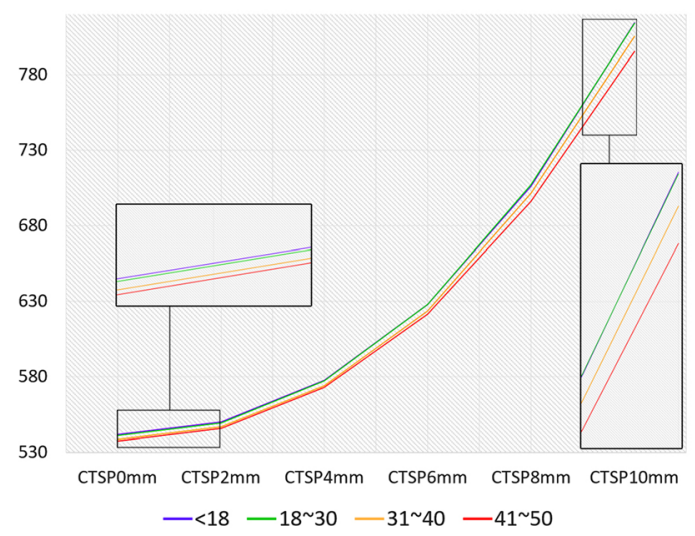

thinning with an increase in age, with increased thinning toward the periphery. CTSP corneal thickness spatial profile

of keratoconus [18]. However, many patients with low risk scores during preoperative screening have developed corneal ectasia after surgery [19-22]. Conversely, some high-risk patients identified during preoperative screening maintain stable vision and a normal corneal morphology for several years after surgery. 
Table 3 Corneal thickness spatial profile $(0-10 \mathrm{~mm})$ for Chinese subjects with healthy eyes stratified into different age groups

\begin{tabular}{lllll}
\hline CTSP $(\mathbf{m m})$ & $<\mathbf{1 8}$ years & $\mathbf{1 8 - 3 0}$ years & $\mathbf{3 1 - 4 0}$ years & $\mathbf{4 1 - 5 0 \text { years }}$ \\
\hline CTSP0mm & $541.53 \pm 30.18$ & $540.76 \pm 29.24$ & $538.58 \pm 28.52$ & $537.24 \pm 28.74$ \\
& $540.00(519.00-560.00)$ & $540.00(521.00-560.00)$ & $538.00(519.00-557.00)$ & $536.50(516.00-557.00)$ \\
CTSP2mm & $549.83 \pm 30.53$ & $549.21 \pm 29.58$ & $546.92 \pm 28.84$ & $545.70 \pm 29.20$ \\
& $548.50(527.50-568.00)$ & $548.00(529.00-568.00)$ & $546.00(527.00-566.00)$ & $544.50(525.00-566.00)$ \\
CTSP4mm & $577.42 \pm 31.69$ & $577.01 \pm 31.26$ & $574.07 \pm 30.06$ & $572.83 \pm 30.66$ \\
& $576.00(556.00-597.00)$ & $576.00(556.00-597.00)$ & $573.00(554.00-593.00)$ & $572.00(551.00-594.00)$ \\
CTSP6mm & $627.79 \pm 34.14$ & $628.00 \pm 33.73$ & $623.74 \pm 32.48$ & $621.58 \pm 33.14$ \\
& $626.00(606.00-650.00)$ & $627.00(606.00-650.00)$ & $623.00(602.00-645.00)$ & $621.00(598.00-644.00)$ \\
CTSP8mm & $706.08 \pm 38.17$ & $707.12 \pm 36.96$ & $701.36 \pm 36.14$ & $695.93 \pm 37.06$ \\
& $703.00(681.00-730.00)$ & $706.00(682.00-731.00)$ & $701.00(677.00-724.00)$ & $695.00(671.00-721.00)$ \\
CTSP10mm & $814.43 \pm 44.69$ & $813.98 \pm 43.42$ & $805.40 \pm 43.34$ & $795.48 \pm 46.60$ \\
& $813.00(784.00-844.00)$ & $813.00(784.00-843.00)$ & $804.00(776.00-834.00)$ & $795.00(764.00-824.00)$ \\
\hline
\end{tabular}

CTSP corneal thickness spatial profile

Values are expressed as means \pm standard deviations or medians (Q1-Q3)

Table 4 Corneal surface height for Chinese subjects with healthy eyes stratified into different age groups

\begin{tabular}{|c|c|c|c|c|c|}
\hline Age groups & $N$ (missing) & Mean \pm SD & M (Q1-Q3) & $\chi^{2}$ & $P$ \\
\hline \multicolumn{6}{|c|}{ Anterior corneal surface height } \\
\hline$<18$ years $^{*}$ & $780(0)$ & $2.10 \pm 1.23$ & $2.00(1.00-3.00)$ & 101.1995 & $<0.0001$ \\
\hline $18-30$ years $^{\triangle}$ & $21,495(1)$ & $2.29 \pm 1.20$ & $2.00(2.00-3.00)$ & & \\
\hline $31-40$ years $^{\triangle}$ & $6945(0)$ & $2.25 \pm 1.32$ & $2.00(1.00-3.00)$ & & \\
\hline $41-50$ years $^{\S}$ & $1398(0)$ & $1.95 \pm 1.45$ & $2.00(1.00-3.00)$ & & \\
\hline \multicolumn{6}{|c|}{ Posterior corneal surface height } \\
\hline$<18$ years $*$ & $780(0)$ & $3.57 \pm 2.69$ & $4.00(2.00-5.00)$ & 142.6874 & $<0.0001$ \\
\hline $18-30$ years* & $21,495(1)$ & $3.75 \pm 2.72$ & $4.00(2.00-5.00)$ & & \\
\hline $31-40$ years ${ }^{\triangle}$ & $6945(0)$ & $3.94 \pm 2.86$ & $4.00(2.00-6.00)$ & & \\
\hline $41-50$ years ${ }^{\S}$ & $1398(0)$ & $4.68 \pm 3.25$ & $5.00(3.00-7.00)$ & & \\
\hline
\end{tabular}

Wilcoxon rank sum test

Different symbols $\left(\triangle,{ }^{*}, \S\right)$ indicate that there are significant differences between groups

$S D$ standard deviation, $M$ median

Many corneal morphological parameters and data provided by corneal topographic maps, including Belin's corneal ectasia analysis parameters, have been formulated via calculations and analyses of collected data according to different principles and algorithms under 
(A) Corneal Surface Height for Different Age Groups

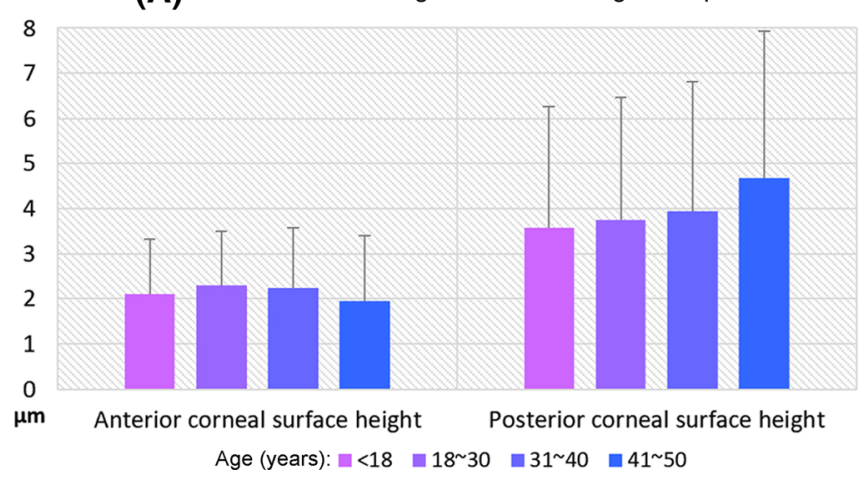

(B) Corneal asymmetry index (IHA, IVA)

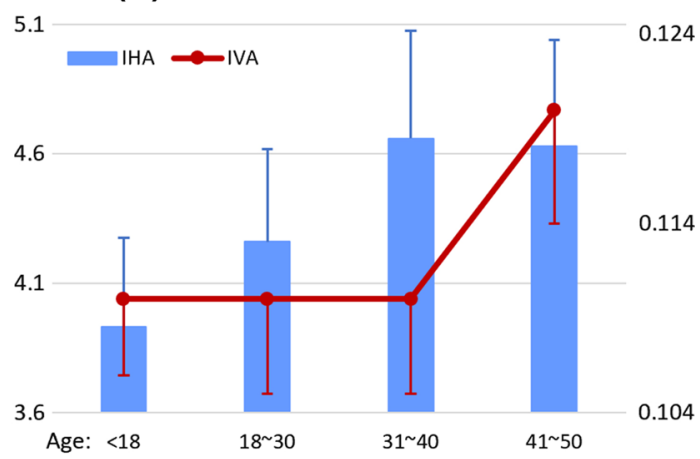

Fig. 4 Age-related trends in the corneal surface height and asymmetry indices in Chinese subjects with healthy eyes. a The anterior corneal surface height exhibits a decrease with age, while the posterior surface height increases with age. b Both IHA and IVA exhibit an increase with age. IHA index of height asymmetry, IVA index of vertical asymmetry

Table 5 IHA and IVA values for Chinese subjects with healthy eyes stratified into different age groups

\begin{tabular}{|c|c|c|c|c|c|}
\hline Age groups & $N$ (missing) & Mean \pm SD & $\mathbf{M}(\mathbf{Q 1}-\mathbf{Q} 3)$ & $\chi^{2}$ & $P$ \\
\hline \multicolumn{6}{|l|}{ IHA } \\
\hline$<18$ years ${ }^{*}$ & $780(0)$ & $3.93 \pm 3.45$ & $3.00(1.35-5.50)$ & 38.2744 & $<0.0001$ \\
\hline $18-30$ years $^{\triangle}$ & $21,495(2)$ & $4.26 \pm 3.65$ & $3.30(1.50-6.00)$ & & \\
\hline $31-40$ years $^{\triangle}$ & $6945(0)$ & $4.66 \pm 4.17$ & $3.50(1.60-6.60)$ & & \\
\hline $41-50$ years $^{\triangle}$ & $1398(0)$ & $4.63 \pm 4.09$ & $3.50(1.50-6.80)$ & & \\
\hline \multicolumn{6}{|l|}{ IVA } \\
\hline$<18$ years ${ }^{*}$ & $780(0)$ & $0.11 \pm 0.04$ & $0.10(0.07-0.13)$ & 10.2398 & 0.0166 \\
\hline $18-30$ years $^{\triangle}$ & $21,495(2)$ & $0.11 \pm 0.05$ & $0.10(0.07-0.14)$ & & \\
\hline $31-40$ years $^{\#}$ & $6945(0)$ & $0.11 \pm 0.05$ & $0.10(0.07-0.14)$ & & \\
\hline $41-50$ years $^{\S}$ & $1398(0)$ & $0.12 \pm 0.06$ & $0.10(0.08-0.14)$ & & \\
\hline
\end{tabular}

Wilcoxon rank sum test

Different symbols $\left(\triangle,{ }^{*}, \S\right)$ indicate significant differences in IHA between groups

$\S$ Indicates significant differences in IVA between groups marked with * or $\triangle$

\# Indicates significant differences in IVA between groups marked with *

$I H A$ index of height asymmetry, $I V A$ index of vertical asymmetry, $S D$ standard deviation, $M$ median

normal value control [23]. The location of the R\&D headquarters for Pentacam in Europe is one of the reasons why most of the normal values used for the analysis of equipment software are based on data for European and American ethnicities. Anthropological studies have shown that Caucasians account for a large proportion of the European and American populations, whereas Mongolian and mixed Mongolian and Caucasian ethnicities account for a large proportion of the Asian population [24]. The corneal morphology varies among regions and populations $[25,26]$; therefore, agerelated analysis of corneal morphological 


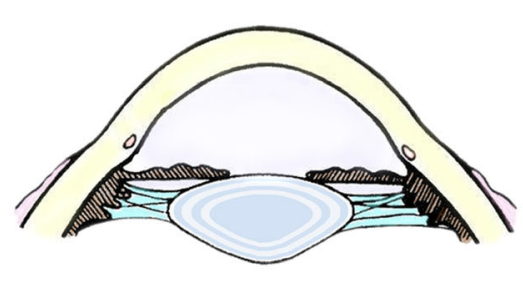

Normal Cornea

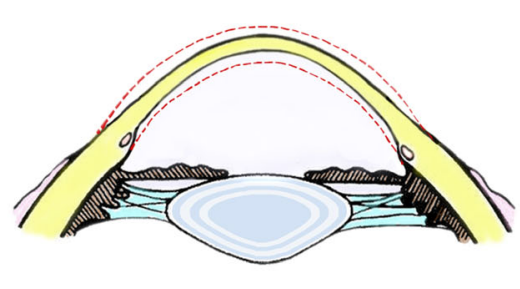

Normal Cornea (with increasing age)

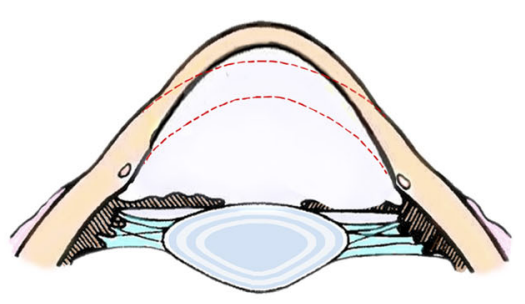

Keratoconus
Fig. 5 Illustration of corneal morphological changes with age in Chinese subjects with healthy eyes. Left to right: normal cornea, normal cornea in aged subjects (overall corneal thinning with an increase in the anterior and

parameters and investigation of age-related changes in these parameters in Asian individuals with healthy eyes may provide more accurate reference data for the early diagnosis of keratoconus in this specific population.

The present multicenter, large-scale study involved five regions of China that are distributed in warm temperate, subtropical, and tropical zones. These regions also show different climatic characteristics, such as an inland climate, a coastal monsoon climate, and an alpine climate, and basically cover the various geographical and inhabitant characteristics of Asia. Thus, comprehensive analysis of data from these regions could represent the corneal morphological characteristics of most Asian populations. By evaluating Chinese subjects with healthy eyes, we found that the corneal morphology gradually changes with age, with an increase in the anterior and posterior corneal curvatures (K1 and $\mathrm{K} 2$ ); a gradual decrease in astigmatism of the anterior and posterior corneal surfaces $(\Delta \mathrm{K})$; a gradual decrease in the central corneal thickness and the thinnest corneal thickness; a decrease in the overall corneal thickness, with a more obvious decrease toward the periphery; a decrease in the anterior corneal surface height and an increase in the posterior corneal surface height; and an increase in IHA and IVA. These findings indicate that the normal corneal morphology does not remain constant and gradually changes from adolescence to middle age. While the cornea becomes thinner toward the periphery, the anterior and posterior corneal curvatures gradually increase. posterior surface curvatures), and keratoconus (true corneal protrusion). The red dotted line represents the original thickness and shape of a normal cornea

These changes result in the transition of the corneal shape from a flat ellipse to an elongated ellipse. However, the anterior and posterior surfaces become smoother with age. Although keratoconus is also characterized by an increase in the anterior and posterior corneal curvatures [27], corneal protrusion, and a decrease in the corneal thickness, the morphological changes associated with this condition are quite different from the age-related changes observed for healthy corneas. A normal cornea gradually shows relative protuberance because of thinning and an increased curvature; however, a cornea affected by keratoconus shows an actual protuberance caused by a change in the corneal structure. Figure 5 clarifies the differences between normal age-related changes in the corneal morphology and pathological changes associated with keratoconus.

A few similar studies have also shown that the corneal curvature increases with age in individuals with healthy eyes [28, 29]. Moreover, similar studies in hundreds of samples have reported that the normal corneal thickness decreases with age [30, 31]. This could be attributed to decreased pressure on the cornea due to aging of the eyelid, muscle relaxation around the eyes, and the effects of gravity over the years. In addition, corneal micromorphological studies have found that the corneal protrusion and thinning observed in keratoconus are caused by a decrease in type I and type IV collagen and the extracellular matrix, the degradation of fibroblasts, the uneven distribution of collagen fibers, and a decrease in 
the number of collagen lamellae [32-34]. Unlike the microscopic changes observed with keratoconus, the fibrous interval of the corneal collagen in healthy eyes decreases with age [35], which probably results in corneal thinning. Other related studies have found that collagen fibers in the corneal stroma of healthy eyes gradually increase with age [36], along with an increase in the corneal tissue hardness [37]. This explains why the biomechanical properties of the cornea remain good despite gradual thinning and relative protrusion. Despite the above results, in fact, for each healthy individual, the change of corneal morphology with age should be very slight, the main corneal parameters remain quite stable in a 40-year span, and very small changes should be expected in the $\mathrm{K}$ readings, and almost none in the thickness or astigmatism magnitude. Owing to very large number of cases, these small differences are statistically significant, despite not being clinically relevant. In fact, the results obtained are consistent with the hypothesis that a normal cornea seems to withstand quite well the effect of IOP and external pressures (blinking, rubbing) just showing a minimal (and not clinically relevant) corneal "steepening" and thinning, most probably due to the natural cross-linking. Thus, clinicians should not expect any noticeable variations in the mean corneal parameters during follow-up of normal patients.

\section{CONCLUSION}

The findings of this study can be used as reference data for the differential diagnosis of subclinical and early keratoconus by refractive surgeons and keratologists treating individuals of Asian descent.

As a result of the limited conditions, the sample capacity and age difference in different regions are indeed the difficulties that we could not overcome in this study. We are contacting more ophthalmic centers in different regions for relevant cooperative research. We will expand the sample size and regions to improve our future research.

\section{ACKNOWLEDGEMENTS}

Funding. This work, including the journal's Rapid Service Fees, was supported by the National Natural Science Foundation of China (grants 82000860, 81873684, 81803333), the Science and Technology Foundation of Tianjin Eye Hospital (grant YKQN1908). These organizations were not involved in the design of the study, collection, analysis, interpretation of data, or writing and publication of the research.

Editorial Assistance. We would like to thank Editage (www.editage.cn) for the English language editing service.

Authorship. All named authors meet the International Committee of Medical Journal Editors (ICMJE) criteria for authorship for this article, take responsibility for the integrity of the work as a whole, and have given their approval for this version to be published.

Authorship Contributions. Ruijue Ma and Yuanyuan Liu were responsible for statistical analysis and writing articles, they contributed equally to the work presented here and should therefore be regarded as equivalent authors. Lin Zhang, Jiaonan Ma and Tong Cui were responsible for data sorting. Yulin Lei, Jie Hou, Zhengwei Shen, Xianglong Yi and Gang Liang were responsible for data collection. Yan Wang was responsible for designing and guiding the whole research.

Disclosures. Ruijue Ma, Yuanyuan Liu, Lin Zhang, Jiaonan Ma, Tong Cui, Yulin Lei, Jie Hou, Zhengwei Shen, Xianglong Yi, Gang Liang, Yan Wang declare that they have no conflicts of interest.

Compliance with Ethics Guidelines. The study protocol adhered to the tenets of the Declaration of Helsinki. Ethical approval was not required because this retrospective study did not deal with human tissue samples, and there were no concerns about privacy. 
Data Availability. The datasets generated and analyzed during the current study are not publicly available at this time as the data also forms part of an ongoing study.

Open Access. This article is licensed under a Creative Commons Attribution-NonCommercial 4.0 International License, which permits any non-commercial use, sharing, adaptation, distribution and reproduction in any medium or format, as long as you give appropriate credit to the original author(s) and the source, provide a link to the Creative Commons licence, and indicate if changes were made. The images or other third party material in this article are included in the article's Creative Commons licence, unless indicated otherwise in a credit line to the material. If material is not included in the article's Creative Commons licence and your intended use is not permitted by statutory regulation or exceeds the permitted use, you will need to obtain permission directly from the copyright holder. To view a copy of this licence, visit http://creativecommons.org/licenses/by$\mathrm{nc} / 4.0 /$.

\section{REFERENCES}

1. Ihalainen A. Clinical and epidemiological features of keratoconus genetic and external factors in the pathogenesis of the disease. Acta Ophthalmol Suppl. 1986;178(178):1-64.

2. Owens H, Gamble G. A profile of keratoconus in New Zealand. Cornea. 2003;22(2):122.

3. Crews MJ, Stern GA. The clinical management of keratoconus: a 6 year retrospective study. CLAO J. 1994;20(3):194.

4. Wojcik KA, Janusz B, Jerzy S, Szaflik JP. Role of biochemical factors in the pathogenesis of keratoconus. Acta Biochim Pol. 2014;61(1):55-62.

5. Godefrooij DA, Wit GAD, Uiterwaal CS, Imhof SM, Wisse RPL. Age-specific incidence and prevalence of keratoconus: a nationwide registration study. Am J Ophthalmol. 2017;175:169-72.

6. Ira C, Jain AK. Between eye asymmetry in keratoconus in an Indian population. Clin Exp Optom. 2010;88(3):146-52.
7. Herber R, Kunert KS, Veliká V, Spoerl E, Pillunat LE, Raiskup F. Influence of the beam profile crosslinking setting on changes in corneal topography and tomography in progressive keratoconus: preliminary results. J Cataract Refract Surg. 2018;44(6): 718-24.

8. Rabinowitz YS. Longitudinal study of the normal eyes in unilateral keratoconus patients. Ophthalmology. 2004;111(3):440-6.

9. Rabinowitz YS. Keratoconus. Surv Ophthalmol. 1998;42(4):297-319.

10. Uçakhan ÖÖ, Çetinkor V, Özkan M, Kanpolat A. Evaluation of Scheimpflug imaging parameters in subclinical keratoconus, keratoconus, and normal eyes. J Cataract Refract Surg. 2011;37(6):1116-24.

11. Vazirani J, Basu S. Keratoconus: current perspectives. Clin Ophthalmol. 2013;2013:2019-30.

12. Gomes JA, Tan D, Rapuano CJ, Belin MW, et al. Global consensus on keratoconus and ectatic diseases. Cornea. 2015;34(4):359.

13. Serdarogullari H, Tetikoglu M, Karahan H, Alin F, Elcioglu M. Prevalence of keratoconus and subclinical keratoconus in subjects with astigmatism using Pentacam derived parameters. J Ophthal Vision Res. 2013;8(3):213-9.

14. Bae GH, Kim JR, Chi HK, Dong HL, Chung ES, Chung TY. Corneal topographic and tomographic analysis of fellow eyes in unilateral keratoconus patients using Pentacam. Am J Ophthalmol. 2014;157(1):103-9.e1.

15. Ruiseñor Vázquez PR, Galletti JD, Minguez N, et al. Pentacam Scheimpflug tomography findings in topographically normal patients and subclinical keratoconus cases. Am J Ophthalmol. 2014;158(1): 32-40.e2.

16. Belin MW, Duncan JK. Keratoconus: the ABCD grading system. Klin Monatsbl Augenheilkd. 2016;233(06):701-7.

17. Mohamed D, Amirul I, Shekar SN, Baird PN. Dominant genetic effects on corneal astigmatism: the genes in myopia (GEM) twin study. Invest Ophthalmol Vis Sci. 2008;49(4):1339.

18. Yan L, Chamberlain W, Ou T, Brass R, Weiss JL, Huang D. Subclinical keratoconus detection bypattern analysis of corneal and epithelial thickness maps with optical coherence tomography. J Cataract Refract Surg. 2016;42(2):284-95.

19. Randleman JB, Woodward M, Lynn MJ, Stulting RD. Risk assessment for ectasia after corneal 
refractive surgery. Ophthalmology. 2008;115(1):3750.e4.

20. Renato A, Dawson DG, Marcella SO, Guerra FP, Caiado ALC, Belin MW. Corneal ectasia after LASIK despite low preoperative risk: tomographic and biomechanical findings in the unoperated, stable, fellow eye. J Refract Surg. 2010;26(11):906-11.

21. Klein SR, Epstein RJ, J Bradley R, R Doyle S. Corneal ectasia after laser in situ keratomileusis in patients without apparent preoperative risk factors. Am J Ophthalmol. 2006;142(4):714.

22. Chan CCK, Christopher H, Gerard S. External analysis of the Randleman Ectasia Risk Factor Score System: a review of 36 cases of post LASIK ectasia. Clin Experiment Ophthalmol. 2010;38(4):335-40.

23. Lopes BT, Ramos IC, Dawson DG, Belin MW. Detection of ectatic corneal diseases based on pentacam. Z Med Phys. 2016;26(2):136-42.

24. Keysertracqui C, Crubézy E, Pamzsav H, Varga T, Ludes B. Population origins in Mongolia: genetic structure analysis of ancient and modern DNA. Am J Phys Anthropol. 2010;131(2):272-81.

25. Sng C, Barton K, Kim H, Yuan S, Budenz DL. Central corneal thickness and its associations with ocular and systemic factors in an Urban West African Population. Am J Ophthalmol. 2016;169:268-75.

26. Ma R, Liu Y, Zhang L, et al. Distribution and trends in corneal thickness parameters in a large population-based multicenter study of young Chinese adults. Invest Ophthalmol Vis Sci. 2018;59(8):3366.

27. Vinciguerra R, Roberts CJ, Azzolini C, Vinciguerra P. Biomechanical characterization of subclinical keratoconus without topographic or tomographic abnormalities. J Refract Surg. 2017;33(6):399.

28. Garzón N, Poyales F, Illarramendi I, et al. Corneal densitometry and its correlation with age, pachymetry, corneal curvature, and refraction. Int Ophthalmol. 2017;37(6):1263-8.
29. Hashemi H, Asgari S, Emamian MH, Mehravaran S, Fotouhi A. Age-related changes in corneal curvature and shape: the Shahroud Eye Cohort Study. Cornea. $2015 ; 34(11): 1456$.

30. Kadhim YJ, Farhood QK. Central corneal thickness of Iraqi population in relation to age, gender, refractive errors, and corneal curvature: a hospitalbased cross-sectional study. Clin Ophthalmol. 2016;10:2369-76.

31. Mwanza JC, Tulenko SE, Herndon LW, et al. Longitudinal change in central corneal thickness in the Tema Eye Survey. Am J Ophthalmol. 2018;186: $10-8$.

32. Collier S. Is the corneal degradation in keratoconus caused by matrix-metalloproteinases? Clin Experim Ophthalmol. 2010;29(6):340-4.

33. Abalain JH, Dossou H, Colin J, Floch HH. Levels of collagen degradation products (telopeptides) in the tear film of patients with keratoconus. Cornea. 2000;19(4):474-6.

34. Romero-Jiménez M, Santodomingo-Rubido J, Wolffsohn JS. Keratoconus: a review. Cont Lens Anterior Eye. 2010;33(4):157-66.

35. Malik NS, Moss SJ, Ahmed N, Furth AJ, Wall RS, Meek KM. Ageing of the human corneal stroma: structural and biochemical changes. Biochem Biophys Acta. 1992;1138(3):222.

36. Daxer A, Misof K, Grabner B, Ettl A, Fratzl P. Collagen fibrils in the human corneal stroma: structure and aging. Invest Ophthalmol Vis Sci. 1998;39(3): 644.

37. Matalia J, Francis M, Tejwani S, Dudeja G, Rajappa $\mathrm{N}$, Sinha RA. Role of age and myopia in simultaneous assessment of corneal and extraocular tissue stiffness by air-puff applanation. J Refract Surg. 2016;32(7):486-93. 1081597X-20160512-02. 